\title{
Bridging the gap
}

\author{
The chiral magnetic exchange interaction, or Dzyaloshinskii-Moriya interaction, is found to propagate through \\ dozens of atomic layers and also to be present in inhomogeneous amorphous alloys. These discoveries extend the \\ parameter space available for realizing magnetic structures with chiral character.
}

\section{Bartel Van Waeyenberge}

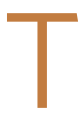
he antisymmetric exchange interaction, or Dzaloshinskii-Moriya interaction, was discovered 60 years ago and explains the weak ferromagnetism observed in antiferromagnetic $\alpha-\mathrm{Fe}_{2} \mathrm{O}_{3}$ crystals $^{1,2}$. It is a superexchange interaction mediated by a non-magnetic atom, and originates from the relativistic spin-orbit interaction. In contrast to the symmetric Heisenberg exchange interaction that favours parallel or antiparallel orientation, the antisymmetric variant favours a canted orientation between neighbouring spins, introducing a chiral character in the magnetic configuration, and supports spin spirals and cycloids, or topological twists such as magnetic skyrmions. Symmetry dictates that such a chiral interaction can only exist if spatial inversion symmetry is broken. In the bulk, this is possible when the crystal structure itself lacks inversion symmetry, so chiral magnetic structures are typically only expected in non-centrosymmetric crystals ${ }^{3}$. However, inversion symmetry is also broken at surfaces or interfaces and it became apparent that the interaction between two magnetic sites at the interface with a non-magnetic material with strong spin-orbit interaction, like a high- $Z$ metal, can have a sizeable chiral contribution in thin magnetic films ${ }^{4}$. This intralayer chiral exchange interaction has boosted the field of chiral magnetism, as it opened new pathways for developing devices based on chiral configurations like magnetic skyrmions ${ }^{5}$.

Now, Dong-Soo Han et al. ${ }^{6}$ and Amalio Fernández-Pacheco et al. ${ }^{7}$ report in Nature Materials their independent observations of a different kind of antisymmetric exchange interaction. They studied the magnetization reversal in multilayer stacks containing two magnetic layers separated by non-magnetic layers and revealed an asymmetry in the switching fields with respect to the direction of the external applied field. This asymmetry cannot be explained by a chiral intralayer interaction that would tilt neighbouring spins within the magnetic layers (Fig. 1a), but requires a canting between the spins in a
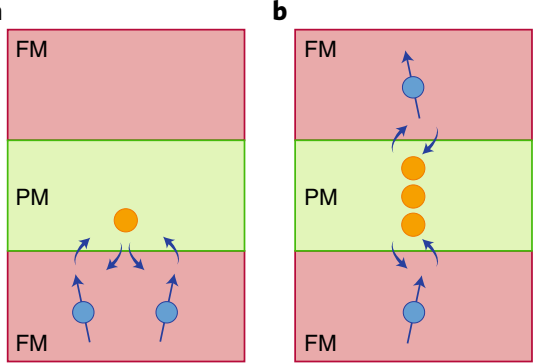

Fig. 1 | Intralayer and interlayer chiral exchange interaction. Sketches illustrating the difference between the two interactions. a, The well-known intralayer or interfacial Dzyaloshinskii-Moriya interaction, which couples two spins (straight blue arrows) of neighbouring sites (blue solid circles) in the ferromagnetic (FM) layer through the interaction at the interface with a paramagnetic metal (PM, yellow solid circles). b. The newly discovered chiral interlayer exchange interaction. Here, two magnetic sites in two different ferromagnetic layers experience an antisymmetric coupling mediated by several atomic layers of paramagnetic metal. The curved arrows represent the exchange interaction between the spins.

the two magnetic layers. This means that a chiral interaction must exist between the two ferromagnetic layers that are separated by several nanometres of non-magnetic material (Fig. 1b). An interaction that is mediated by tens of atomic layers must be an indirect one, similar to the RudermanKittel-Kasuya-Yosida (RKKY) interaction between localized spins mediated by conduction electrons ${ }^{8-10}$.

The discovery that another antisymmetric chiral exchange interaction, besides the symmetric RKKY interaction, can bridge many atomic layers of a normal paramagnetic metal is surprising. The observed coupling in these experiments is small but significant, and further research will be required to find out the details of the distance dependence of this interaction. It will have to be taken into account when designing magnetic multilayer systems and, like any of the chiral interactions, will require that the correct symmetries are broken in order to exploit this interaction.

This notion of broken symmetry links to a related work published in Nature Materials, in which Duck-Ho Kim and colleagues ${ }^{11}$ reveal another aspect of the chiral exchange interaction. They argue that a non-centrosymmetric crystal structure, or even an ordered structure, is not required to realize a bulk Dzyaloshinskii-Moriya interaction, but that a breaking of the spatial symmetry of the composition can be sufficient. Their experiments on amorphous ferrimagnetic films reveal that the interaction strength is proportional to a gradient in the composition, directly illustrating the necessary condition for this chiral interaction. This contribution makes it clear that any breaking of the inversion symmetry combined with the spin-orbit interaction can lead to chiral magnetism, be it structural, geometrical or compositional. This adds one more degree of freedom for the design of new chiral magnets.

Taken together, these works provide new possibilities to create chiral materials that can be used to design functional magnetic devices that are promising for future nonvolatile memory and logic technologies.

Bartel Van Waeyenberge

Department of Solid State Sciences, Ghent University, Ghent, Belgium.

e-mail:bartel.vanwaeyenberge@ugent.be

Published online: 19 June 2019

https://doi.org/10.1038/s41563-019-0411-7

\footnotetext{
References

1. Dzyaloshinskii, I. J. Phys. Chem. Solids 4, 241-255 (1958).

2. Moriya, T. Phys. Rev. 120, 91-98 (1960).

3. Mühlbauer, S. et al. Science 323, 915-919 (2009).

4. Bode, M. et al. Nature 447, 190-193 (2007).

5. Heinze, S. et al. Nat. Phys. 7, 713-718 (2011)

6. Han, D.-S. et al. Nat. Mater. https://doi.org/10.1038/s41563-0190370-z (2019).

7. Fernández-Pacheco, A. et al. Nat. Mater. https://doi.org/10.1038/ s41563-019-0386-4 (2019)

8. Ruderman, M. A. \& Kittel, C. Phys. Rev. 96, 99-102 (1954).

9. Kasuya, T. Prog. Theor. Phys. 16, 45-57 (1956).

10. Yosida, K. Phys. Rev. 106, 893-898 (1957)

11. Kim, D.-H. et al. Nat. Mater. https://doi.org/10.1038/s41563-0190380-x (2019).
} 